REVISTA

EPUEACIÓN
Revista Educación

ISSN: 0379-7082

ISSN: 2215-2644

revedu@gmail.com

Universidad de Costa Rica

Costa Rica

\title{
Exploración sobre la oferta de los posgrados en Economía Social en los países hispanohablantes
}

de la O, Dyalá; Fedorov, Andrei N; Urra Urbieta, J. Anastasio; Arzadun, Paula

Exploración sobre la oferta de los posgrados en Economía Social en los países hispanohablantes

Revista Educación, vol. 43, núm. 2, 2019

Universidad de Costa Rica, Costa Rica

Disponible en: http://www.redalyc.org/articulo.oa?id=44058158012

DOI: https://doi.org/10.15517/revedu.v43i2.30706

Esta obra está bajo una Licencia Creative Commons Atribución-NoComercial-SinDerivar 3.0 Internacional. 


\title{
Exploración sobre la oferta de los posgrados en Economía Social en los países hispanohablantes
}

\author{
Exploring Postgraduate Socioeconomics Programs in Spanish-Speaking Countries
}

Dyalá de la $O$

Instituto Tecnológico de Costa Rica, Costa Rica

ddelao@tec.ac.cr

iD http://orcid.org/0000-0002-2974-5554

Andrei N Fedorov

Instituto Tecnológico de Costa Rica, Costa Rica

afedorov@tec.ac.cr

(iD) http://orcid.org/0000-0001-7679-2321

\section{J. Anastasio Urra Urbieta}

Universidad de Valencia, España

anastasio.urra@uv.es

(iD) http://orcid.org/0000-0001-7679-2321

Paula Arzadun

Instituto Tecnológico de Costa Rica, Costa Rica

parzadun@tec.ac.cr

iD http://orcid.org/0000-0002-9241-0255

\author{
DOI: https://doi.org/10.15517/revedu.v43i2.30706 \\ Redalyc: http://www.redalyc.org/articulo.oa?id=44058158012
}

Des:/orcid.org/0000-0002-9241-0255

Recepción: 10 Octubre 2017

Aprobación: 08 Mayo 2019

\section{Resumen:}

En los últimos años las actividades sin fines de lucro han sido promovidas como un mecanismo alternativo para el desarrollo en el contexto de la situación económica mundial. Sin embargo, a pesar de sus potencialidades, esas actividades continúan siendo relativamente poco expandidas y requieren de ampliar los conocimientos sobre ellas. En este sentido la universidad puede convertirse en un generador y transmisor de saberes sobre el tema de economía social. Por esta razón, se plantea desarrollar un análisis de la oferta de programas de posgrado en Economía Social en las instituciones de educación superior latinoamericanas y españolas. El artículo presenta el proceso y los resultados de una pesquisa exploratoria que permitió determinar el interés de las universidades en brindar opciones de formación de alto nivel académico y profesional en torno al tema del desarrollo económico más inclusivo y equitativo de las comunidades y naciones, y conocer las características curriculares fundamentales de los programas de maestría enfocados en esta temática. Este artículo puede ser de interés de los especialistas en economía, gestores de las políticas públicas, académicos, curriculistas y autoridades universitarias.

Palabras Clave: Economía Social, Programas de Posgrado, Currículo Universitario.

\section{Abstract:}

Nonprofit activities have garnered much attention in the past few years as an alternative development method for the global economy. However, despite its potentials, these activities are relatively unknown and have a limited outreach. Academia help resolve this knowledge gap and help generate and convey a better understanding about these activities. One way is to develop postgraduate Socioeconomics programs in Latin America and Spain. This is an exploratory study that gauges interest among universities in offering a graduate program option in Socioeconomics, an area of Economics aimed at a more inclusive and fair economic development of communities and nations. The article also considers curricular aspects required for a Master's program in this area of interest. This study may be of interest to economists and policy-makers, academics, curriculum specialists and university authorities. 
KEYWORDs: Social Economics, Postgraduate Education, University Curriculum.

\section{INTRODUCCIÓN}

La economía social consiste en actividades sin fines de lucro, presentes en todos los sectores económicos, las cuales incluso suelen atender necesidades y regiones relegadas por el estado o que carecen de interés para el sector privado por motivos de rentabilidad (Molloy, McFeeley y Connolly, 1999). Actualmente es considerada como un nuevo paradigma ante la corriente capitalista (Aguilar, 2016). Más concretamente, y de acuerdo con el Primer Congreso de la Economía Social, que se efectuó en España el 11 de diciembre de 1993, la economía social está formada por toda actividad económica, basada en la asociación de personas en entidades de tipo democrático y participativo, con primacía de las aportaciones personales y de trabajo sobre el capital. Si bien las experiencias de economía social encuentran antecedentes desde el siglo XIX, han sido especialmente impulsadas en los últimos años gracias a la búsqueda de respuestas alternativas a la crítica situación derivada del neoliberalismo, que ha llevado a la exclusión masiva del empleo, el desgaste de los salarios y de los derechos sociales, la concentración ampliada de la riqueza y la ferocidad de los mercados globalizados (Coraggio, 2011).

En estos tiempos, cuando el sistema económico no da ninguna salida al género humano, surge como una alternativa exitosa en la historia, con algunas dificultades muy importantes para avanzar, pero con logros muy significativos, la economía social (Kliksberg, 2002; Sen y Kliksberg, 2009; Coraggio, 2014). Así, las empresas sociales se encuentran en una posición de desventaja desde el punto de vista competitivo, por lo tanto, para enfrentar este reto requieren de alianzas estratégicas como acuerdos de dos o más partes para la consecución conjunta de objetivos

El derrumbe de la economía hegemónica mundial está dejando muchas víctimas, millones de desempleados y miríadas de personas en situación de vulnerabilidad. Para poder continuar con la búsqueda del éxito económico, equilibrado con la equidad y la justicia social, desde la escala local hasta la mundial, se deberá brindar apoyo desde la política pública para que las empresas sociales logren manejar sus limitaciones y afrontar los problemas que se les presentan (Kliksberg, 2002; Sen y Kliksberg, 2009).

La economía social tiene potencialidades como forma de organización productiva y, por su carácter estratégico defensivo marginal, adopta funciones transformadoras de gestión, inserción y movilidad social, educación y formación en valores. De tal modo, para alcanzar el éxito económico, equilibrado y justo, la economía social pareciera ser la forma mejor dispuesta, la cual, sin embargo, se requiere de condiciones subjetivas y cambios institucionales que la promuevan e impulsen (Pedreño, 2017; Tomás, 1997).

En este sentido, la universidad puede aportar mediante incorporación de estos temas en los planes de estudio y a través de la generación de conocimiento para poder comprender y ampliar los saberes en economía social, proponiendo su marco conceptual y operacional. Por lo tanto, el presente estudio establece como objetivo explorar la oferta de los programas de posgrado en Economía Social que existe en los países hispanoparlantes de América y Europa y comparar estos programas desde la perspectiva de los componentes fundamentales de sus currículos.

\section{REVISIÓN DE LITERATURA}

La economía social surgió a mediados del siglo XIX como reacción al capitalismo irracional de la Revolución Industrial. Las ideas de Fourier, Owen, Saint-Simón y otros, llevaron a desarrollar la base de lo que hoy se conoce como economía social, cuyo objetivo es producir para ganarse la vida, en comunión con sus semejantes, haciéndose cargo de los excluidos, sin sobreexplotación, ni destrucción del medio ambiente. Esta economía propulsa la auténtica revolución social, pues provee de manera sostenible las bases materiales para 
el desarrollo personal, material y ambiental del ser humano (Kliksberg, 2002; Sen y Kliksberg, 2009; Gómez, 2013; Gómez y Gómez, 2014; García y Ballabrera, 2015).

Las iniciativas de economía social se basan en principios relacionados especialmente con las necesidades de las personas. Se trata de una cooperación eficaz, interdependiente y con la participación activa de los ciudadanos, para poder satisfacer las necesidades sociales y económicas de las comunidades. Tiene que ver con la creación de una sociedad plenamente democrática, que promueve la justicia social, la igualdad fundamental y la igualdad de oportunidades (Chaves y Monzón, 2018; Molloy et al., 1999).

La aceptación del concepto economía social varía según la región. Mientras que, para las autoridades públicas, las empresas de la economía social y los académicos de Hispanoamérica y del Norte de África la aceptación del concepto es escasa o nula, en Europa este es de aceptación moderada de parte de los tres grupos de actores (Chaves, Monzón, Pérez y Radrigán, 2013).

Entre los países hispanoparlantes de América, se destacan Colombia y Honduras, por ser los únicos de la región, donde las autoridades muestran una alta aceptación al concepto. Asimismo, destacan las empresas sociales colombianas, al ser las únicas con una elevada aceptación al concepto. Del mismo modo, las autoridades públicas y las empresas de la economía social de Marruecos y Túnez indican una elevada aceptación al concepto. Sin embargo, ahí no se evidencia la aceptación del mismo por parte del mundo académico. Finalmente, España es el único país de Europa donde la aceptación del concepto es elevada por parte de todos los sectores: autoridades públicas, empresas de la economía social y los académicos (Chaves et al., 2013). Estos datos se resumen en la Tabla 1.

\section{TABLA1 \\ Aceptación del concepto economía social}

\begin{tabular}{|c|c|c|c|c|c|c|c|c|c|}
\hline \multirow[b]{2}{*}{$\%$} & \multicolumn{3}{|c|}{ Latinoamérica } & \multicolumn{3}{|l|}{ Europa } & \multicolumn{3}{|c|}{ Norte de $\mathrm{A}$ frica } \\
\hline & $\begin{array}{c}\text { Escaso } \\
\text { I Nulo }\end{array}$ & Moderado & Elevado & $\begin{array}{l}\text { Escaso } \\
\text { I } \\
\text { Nulo }\end{array}$ & Moderado & Elevado & $\begin{array}{l}\text { Escaso } \\
\text { / Nulo }\end{array}$ & Moderado & Elevado \\
\hline $\begin{array}{l}\text { Por las } \\
\text { autoridades } \\
\text { públicas }\end{array}$ & 76.47 & 5.88 & 17.65 & 37.93 & 55.17 & 6.90 & 50.00 & .00 & 50.00 \\
\hline $\begin{array}{l}\text { Por las } \\
\text { empresas } \\
\text { de la } \\
\text { economía } \\
\text { social }\end{array}$ & 76.47 & 11.76 & 11.76 & 24.14 & 58.62 & 17.24 & 50.00 & .00 & 50.00 \\
\hline $\begin{array}{l}\text { Por el } \\
\text { mundo } \\
\text { académico } \\
\text { - científico }\end{array}$ & 64.71 & 35.29 & .00 & 27.59 & 65.52 & 6.90 & 100.00 & .00 & .00 \\
\hline
\end{tabular}

Fuente: elaboración propia, con base en Chaves et al. (2013).

Cabe resaltar que cuando se hace referencia a la economía social, no existe precisión. Mientras unos incluyen aquellas organizaciones económicas sin fines de lucro, otros amplían su alcance al considerar organizaciones sociales y políticas que no poseen misión y naturaleza económica (Tomás, 1997). Existe evidencia sobre la aceptación de conceptos relacionados, como lo son cooperativas, empresas sociales, empresas del sector no lucrativo y empresas del tercer sector (Chaves et al., 2013). En Hispanoamérica el concepto más aceptado es el de cooperativas, en Europa lo es el sector no lucrativo (ONG) y en el Norte de África son las empresas del tercer sector (ver Tabla 2). 
TABLA 2

Aceptación de otros conceptos de la economía social

\begin{tabular}{|c|c|c|c|c|c|c|c|c|c|}
\hline \multirow{3}{*}{$\%$} & \multicolumn{3}{|c|}{ Latinoamérica } & \multicolumn{3}{|l|}{ Europa } & \multicolumn{3}{|c|}{ Norte de $A$ frica } \\
\hline & Escaso & & & Escaso & & & Escaso & & \\
\hline & I & Moderado & Elevado & I & Moderado & Elevado & I & Moderado & Elevado \\
\hline Cooperativas & 5.88 & .00 & 94.12 & NA & NA & NA & .00 & .00 & 100.00 \\
\hline $\begin{array}{l}\text { Empresas } \\
\text { sociales }\end{array}$ & 70.59 & .00 & 29.41 & 34.48 & 51.72 & 13.79 & .00 & .00 & 100.00 \\
\hline Sector no & & & & & & & & & \\
\hline $\begin{array}{l}\text { lucrativo } \\
\text { (ONG) }\end{array}$ & 76.47 & 11.76 & 11.76 & 6.90 & 55.17 & 37.93 & .00 & .00 & 100.00 \\
\hline Tercer sector & 88.24 & 5.88 & 5.88 & 34.48 & 37.93 & 27.59 & .00 & .00 & 100.00 \\
\hline
\end{tabular}

Fuente: elaboración propia, con base en Chaves et al. (2013).

La economía social se define en dos vertientes: la de mercado y la de no mercado, que se muestran en la Figura 1. En ambas vertientes las empresas son organizadas formalmente, con autonomía de decisión, libertad de adhesión y buscan satisfacer las necesidades de los socios. La vertiente de mercado produce bienes y servicios y la toma de decisiones ahí no está ligada a los aportes de capital, mientras que, en la de no mercado se producen servicios a favor de la familia y los excedentes no son de apropiación de los agentes económicos (Chaves et al., 2013; Pérez de Mendiguren, Etxezarreta y Guridi, 2008).

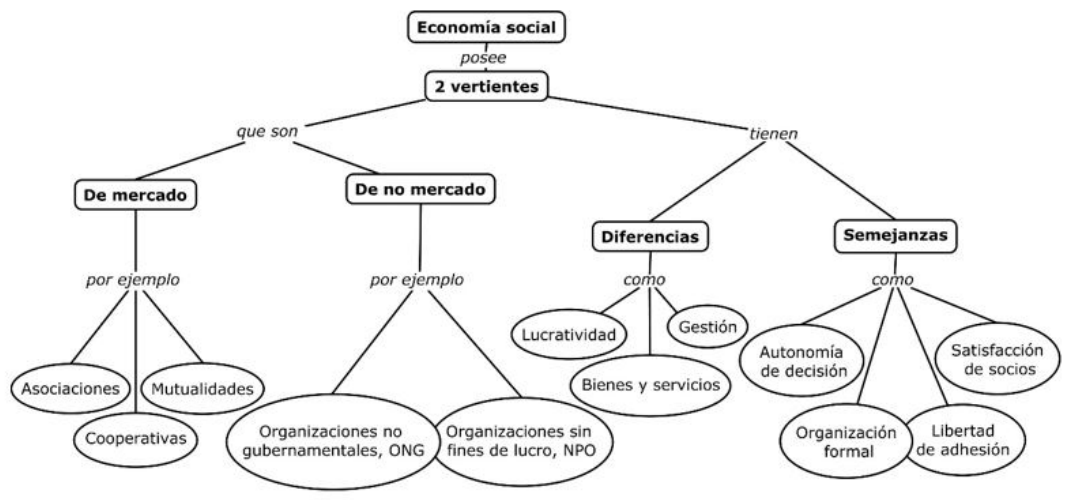

FIGURA 1

Dos vertientes de la economía social

Fuente: Elaboración propia

Por otro lado, la revisión de la literatura evidencia que del tronco común de la economía social surgen la economía solidaria (Europa y Latinoamérica), la economía popular o economía del trabajo o economía social y solidaria (Suramérica, en particular Argentina), desarrollo social (México) y la empresa social (Estados Unidos). No obstante, las dos corrientes más fuertes son la conceptualización europea y la conceptualización latinoamericana, que, si bien coinciden en puntos importantes, también presentan algunas diferencias (Pérez de Mendiguren et al., 2008; Chaves et al., 2013). Las convergencias y divergencias en la conceptualización latinoamericana y europea de la economía social se resumen por medio de la Figura 2. 


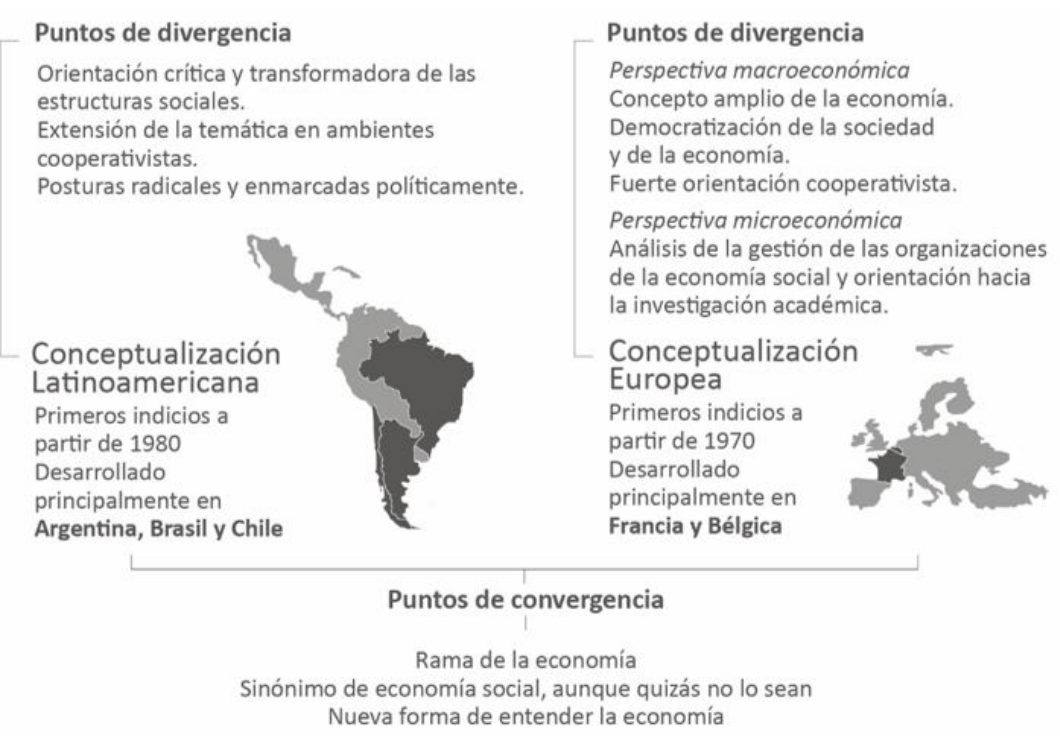

FIGURA 2

Economía social: conceptualización latinoamericana y europea. Fuente: elaboración propia. Fuente: Elaboración propia

La economía social, como corriente ideológica de organización económica, genera empleo y fomenta el espíritu empresarial mediante el cumplimiento de las necesidades sociales y, muy a menudo, mediante la inclusión de las personas excluidas socialmente. Constituye una amplia gama de actividades que tienen el potencial de proporcionar oportunidades para que las personas y las comunidades locales puedan participar en todas las etapas del proceso de regeneración económica local y la creación de empleo, desde la identificación de las necesidades básicas, hasta la puesta en marcha de iniciativas (Molloy et al., 1999; Amin, Cameron y Hudson, 2004).

Que la economía sea percibida desde un enfoque social debería resultar innecesario pues, de hecho, la economía siempre ha sido una ciencia social. No obstante, este enfoque explícito responde a la necesidad de recuperar lo que el capitalismo ha arrebatado a la economía, ya que su desarrollo ha mantenido una relación inversamente proporcional con el de la justicia y la equidad económica, social, cultural y ambiental (Piketty, 2014). Asimismo, en los últimos tiempos han resurgido o se han renovado una serie de nombres, prácticas, criterios y sentidos que se buscan mutuamente $^{[1]}$ y que hacen referencia al valor básico de la economía: la solidaridad (Coraggio, 2011).

En relación con estos resurgimientos y renovaciones, uno de los grandes retos que deben enfrentar las universidades en los países de habla hispana, es enfocar la enseñanza de la economía en lo que la sociedad hoy en día demanda de los economistas y de otros profesionales en ciencias económicas. Un profesional sólidamente formado requiere de herramientas analíticas y de la comprensión de las realidades económicas, sociales e institucionales de su entorno (Lora y Ñopo, 2009). Sin embargo, la adquisición de estas cualidades por los economistas depende, en gran medida, de los planes de estudio que hayan seguido durante su formación universitaria. Por lo tanto, para contribuir a comprender cómo se lleva a cabo el análisis económico de la región, es necesario estudiar el currículo de especialización de los economistas y otros profesionales afines, que son responsables del perfeccionamiento de los programas de desarrollo económico local.

Existen diversos estudios y propuestas para la definición del término currículo, entre las cuales destacan aquellas delimitadas a las experiencias diseñadas para la población estudiantil, las restringidas a las estructuras de resultados basadas en el aprendizaje y las determinadas por todas las experiencias que el estudiantado lleva 
a cabo bajo la tutela de una institución educativa. Algunos de estos estudios y propuestas tratan de definir un currículo oculto, mientras que otros prefieren limitar sus definiciones al currículo oficial (Kemmis, 1998).

El currículo es un intento de comunicar los principios esenciales de una propuesta educativa de tal forma que quede abierta al escrutinio crítico y pueda ser traducida efectivamente a la práctica (Stenhouse, 2003).En relación con esta perspectiva, el currículo es el puente entre la teoría y la práctica, que permite una estructura flexible, adaptada al medio y pertinente con la realidad, vinculado de forma coherente en ciclos, áreas, cursos, proyectos y sistema evaluativo (Gimeno, 1991).

Siguiendo la tradición y la práctica local, se utiliza el vocablo currículo como sinónimo del término plan de estudios. La composición de un plan de estudios o currículo de un programa de posgrado se explica por medio de la Figura 3.

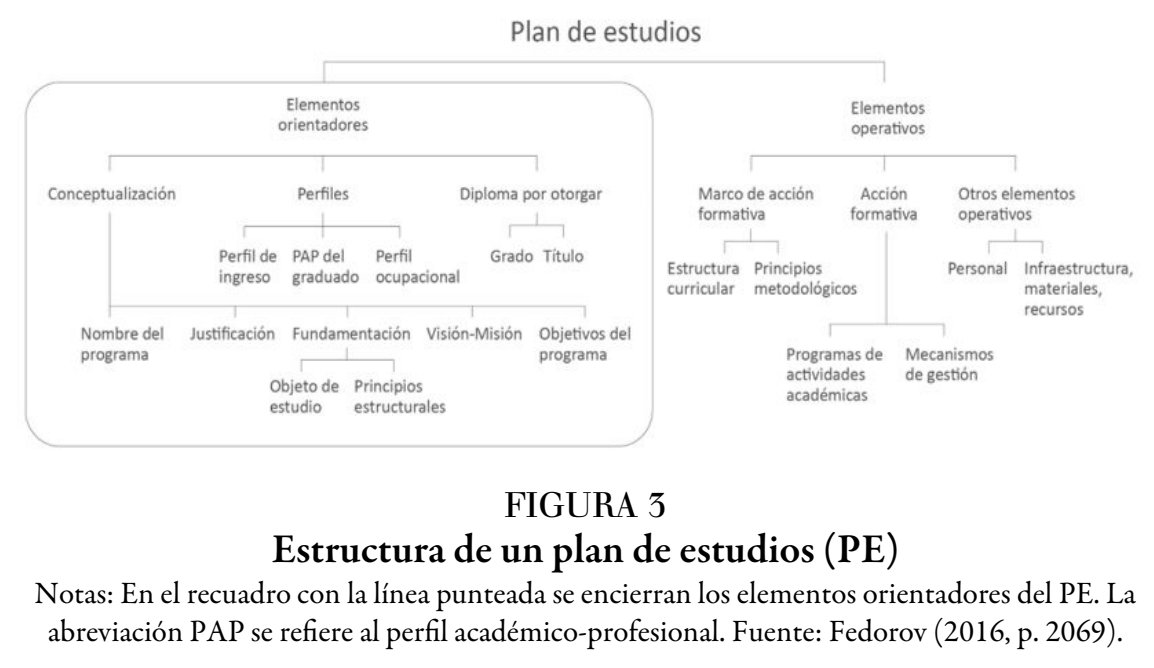

En la mayoría de las universidades del mundo, el currículo de los programas de posgrado, que se encargan de formar profesionales de alto nivel, destinados a liderar las empresas y organizaciones actuales, se basa en el paradigma de la economía ortodoxa, asociada con la economía neoclásica y keynesiana. Esta corriente ideológica se enfoca en el estudio de los recursos, la creación de riqueza y la producción, distribución y consumo de bienes y servicios, para la satisfacción de las necesidades humanas; es decir, analiza las decisiones relacionadas entre la disposición de recursos y la jerarquización de necesidades de los individuos (Samuelson y Nordhaus, 2001).

La formación profesional debe brindar no solo los insumos necesarios para la obtención de un título, sino que además debe ofrecer las bases para incorporar los conocimientos adquiridos a la práctica y facilitar la adaptación de los mismos al contexto (Chaverri y Sanabria, 2010). Además, la enseñanza de la economía no está otorgando respuesta a las interrogantes actuales (Guin y Vidal, 2015). La sociedad requiere de economistas y otros profesionales líderes, formados con un alto nivel teórico y práctico, con el dominio del paradigma completo de la economía, incluyendo la economía social. Esta tarea la debe asumir la universidad, en parte por medio de los programas de alto nivel, como las maestrías académicas y profesionales.

\section{Metodología}

La presente pesquisa se llevó a cabo durante el primer semestre de 2016 y se situó en el dominio de la investigación educativa, específicamente en el área del desarrollo curricular en la enseñanza de la economía, mientras que su alcance fue de carácter exploratorio. El objeto de este trabajo consistió en ejecutar un primer sondeo de la oferta de programas de maestría en Economía Social de las universidades españolas y las de los países latinoamericanos de habla hispana, y un análisis comparativo de los elementos fundamentales u orientadores de sus respectivos currículos. 
A partir de la búsqueda y triangulación por Internet, se logró identificar un total de 39 programas de maestría, ofertados en Latinoamérica y España. Sin embargo, se descartaron cuatro de ellos, pues si bien aparecieron dentro de la oferta académica de las universidades correspondientes, la información por medio de los hipervínculos no estaba disponible. Con base en la información, obtenida de las páginas web, se organizaron y se clasificaron los datos para llevar a cabo un análisis de las ofertas.

La comparación de los currículos de las maestrías relacionadas con la economía social se basó en el análisis de los elementos orientadores de sus planes de estudio. El plan de estudios (PE) es un documento curricular que contiene la previsión y ordenamiento detallado y metódico del ámbito académico y administrativo de un determinado programa universitario. Incluye una serie de componentes, definidos en correspondencia con las regulaciones curriculares vigentes en cada institución y sistema educativo. Estos componentes, según su nivel de inclusión, pueden ser catalogados como elementos orientadores o elementos operativos (ver Figura 3).

Entre los elementos orientadores se encuentran los siguientes: conceptualización, perfiles y diploma por otorgar. La conceptualización, por su lado, incluye los componentes curriculares como el nombre del programa, la justificación, la fundamentación (que incorpora la definición del objeto de estudio y los principios curriculares), los objetivos del programa visto como una organización, la visión y la misión. Los perfiles se subdividen en el perfil de ingreso, el perfil académico-profesional del graduado y él ocupacional. Mientras tanto, el diploma por otorgar incorpora los datos relativos al grado y el título del respectivo posgrado (Fedorov, 2016).

La información recopilada sobre los programas de maestría en Economía Social permitió elaborar la comparación de los siguientes elementos curriculares: objeto de estudio, diploma por otorgar, justificación, objetivos del programa, perfiles de ingreso, egreso y de ocupación.

\section{Resultados}

a exploración efectuada mostró que en España y Latinoamérica hispanohablante la oferta de posgrados, donde se otorga un diploma con el grado académico de maestría y el título relativo a la Economía Social o a alguna de las variaciones expuestas por Coraggio (2011), la compone un total de 35 programas. Se reveló que España es el país donde más se ofrece este tipo de formación, con una oferta de 7 programas, seguido por México con 6 programas y Colombia con 5. Es importante señalar que en tan solo 9 de 19 países hispanoamericanos existen este tipo de opciones de formación académico-profesional. La cantidad de ofertas de este tipo de maestrías por país se muestra en la Figura 4. 


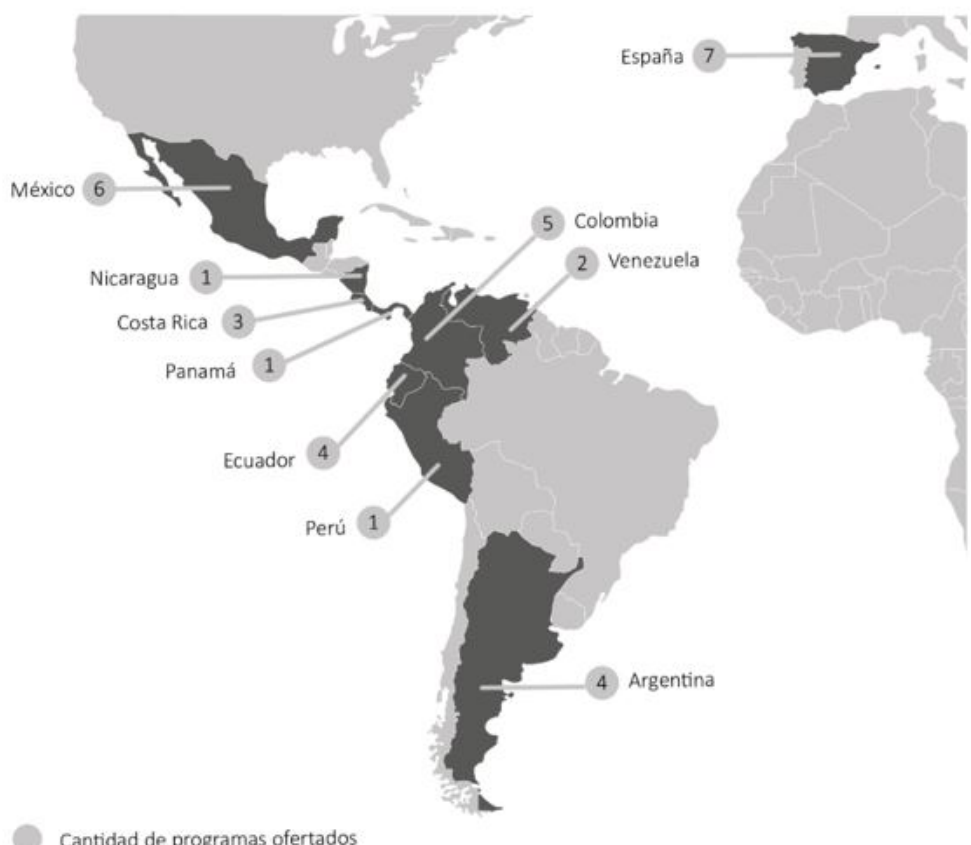

Cantidad de programas ofertados

FIGURA 4

Cantidad de programas de maestría en economía social en España y países hispanohablantes de Latinoamérica

Fuente: Elaboración propia

De acuerdo con los resultados del estudio, las justificaciones, brindadas por las universidades para ofertar este tipo de programas de posgrado, giraron en torno a una serie de necesidades detectadas en su entorno como, por ejemplo, la necesidad de formación de nuevos profesionales e investigadores, la importancia de construir nuevas formas de organización económica y la búsqueda de respuestas a la economía de globalización.

La Universidad Nacional de General Sarmiento, en Argentina, expone la siguiente justificación de su maestría en Economía Social:

Crear un enfoque alternativo a los programas de enseñanza, basados en la economía ortodoxa neoclásica, que contribuya con el desarrollo de un nuevo campo académico y profesional multidisciplinario, que pueda dar cuenta de las nuevas formas de organización económica en un proceso de construcción de la otra economía. (Universidad Nacional de General Sarmiento, 2019).

La Facultad Latinoamericana de Ciencias Sociales (FLACSO), con sede en Argentina, brinda la siguiente justificación: "Fortalecer los sectores más vulnerables en lo social y en lo productivo, bajo nuevos enfoques, presentándose como un cambio de ciclo a nivel político, económico y de modelo de desarrollo" (FLACSO Argentina, 2019). Mientras que la FLACSO con sede en México justifica su oferta así:

Dar respuesta a los problemas generados por la economía global, desde una visión ética, reforzando los conocimientos de la actividad empresarial, con la dimensión demográfica, en su articulación con los complejos procesos económicos, sociales, políticos y culturales, que estructuran la dinámica de las poblaciones. (FLACSO México, s.f.).

La Universidad Nacional de General Sarmiento, en Argentina, expone la siguiente justificación de su maestría en economía social: "Contribuir al desarrollo de un amplio sector orgánico de Economía Social y Solidaria formando profesionales especializados y experimentados capacitados para tal fin" (Universidad Nacional de General Sarmiento, 2019, párr. 1). La Facultad Latinoamericana de Ciencias Sociales 
(FLACSO), con sede en Argentina, ofrece la siguiente justificación: "brindar una formación de excelencia a profesionales que se desempeñan en el sector público, social y privado, y mejorar las capacidades de gestión de las políticas públicas" (FLACSO Argentina, 2019, párr. 4). Mientras que la FLACSO con sede en México justifica su oferta así: "Preparar expertos capaces de contribuir a la elaboración de diagnósticos, definición de políticas y diseño de programas en las áreas temáticas mencionadas, y de incidir sobre los problemas que enfrentan las sociedades latinoamericanas" (FLACSO México, s.f., párr. 2). La Universidad Central de Venezuela declara que su maestría en el área de la economía social se justifica por la necesidad de "formar investigadores y profesionales reflexivos, con una perspectiva integral e interdisciplinaria sobre el desarrollo nacional, regional y local" (Universidad Central de Venezuela, 2016, párr. 2). Mientras tanto, la Pontificia Universidad Católica del Ecuador considera que el programa está diseñado para formar "profesionales con competencias desarrolladas para la investigación, diseño, gestión y evaluación de programas y proyectos sociales, económicos y ambientales, generados con la participación del talento humano de los actores sociales e institucionales, del desarrollo local y comunitario." (Pontificia Universidad Católica del Ecuador, 2014, pp. 2 y 3$)$.

En relación con el objeto de estudio, se determinó que este responde a las necesidades y cambios en la realidad social; enfocándose en la comprensión de la gestión y modernización del estado (enfoque macroeconómico), el estudio de fenómenos y procesos sociales y su relación con el estado, la potencialización de capacidades de gestión para el bienestar social y el uso racional, equilibrado y sostenible de los recursos naturales.

En relación con los objetivos principales de los programas ofertados, se detectó que estos, en términos generales, giran en torno a la idea de brindar una formación teórica y metodológica para el análisis, comprensión y conocimiento del modo de intervención de la política pública y de las organizaciones de la sociedad en el campo de la economía social. No obstante, se observó una variación en este sentido entre los programas: unas se orientan más hacia la economía social y otras hacia el desarrollo territorial.

Aquellos programas del enfoque de la economía social pretenden alcanzar la formación plural que permite generar conocimientos y adquirir una visión integrada de la economía y de la sociedad. Estas maestrías profundizan en los aspectos específicos relativos a la economía social y su dinámica, y pretenden formar profesionales con conocimientos, habilidades y actitudes que les permitan gestionar programas y proyectos fundados en la concepción de la economía social.

Mientras que los programas enfocados más en el desarrollo territorial ofrecen la formación teóricametodológica sobre desarrollo local o territorial y economía social, profundizan en los conocimientos científicos, teóricos y saberes populares propios que soportan el desarrollo político, económico, ambiental y social de la vida territorial. Buscan el desarrollo de competencias para el manejo de la empresa cooperativa como organización solidaria y su articulación con el desarrollo territorial, equitativo y sostenible.

Se observó que en ambas corrientes los programas estudian cuestiones jurídicas, económicas, políticas y sociológicas, vinculadas a la problemática de las entidades de la economía social y del desarrollo territorial. Se orientan a la formación de profesionales e investigadores especializados en la gestión de las entidades de la economía social (de mercado y de no mercado), aportando a los participantes la capacidad suficiente para analizar y evaluar los aspectos empresariales, desde el punto de vista jurídico, fiscal, contable-financiero, organizativo, comercial, estratégico y de gestión de capital humano, entre otras. En la Figura 5 se reflejan las principales áreas temáticas, incluidas en el plan de estudios de los programas analizados. 


\begin{tabular}{|c|c|c|c|}
\hline \multicolumn{4}{|c|}{ Economía Social } \\
\hline Jurídica & Económica & Política & Psicológica \\
\hline
\end{tabular}

\section{FIGURA 5}

Áreas curriculares de los programas analizados.

Fuente: Elaboración propia

El análisis de los perfiles de ingreso reveló que el 60\% de las ofertas se destina a profesionales con un grado universitario de bachillerato o licenciatura y el título en cualquier área del conocimiento, relacionada con las organizaciones sociales, las cuales aspiran a convertirse en agentes de cambio social y económico; o bien, con interés en una formación que les permita participar en la gestión de planes, programas y proyectos de desarrollo local y comunitario. Un 30\% se orienta hacia los profesionales con el diploma de grado en cualquier área de conocimiento, pero con características específicas, como técnicos, directivos y empresarios, que trabajan en la gestión social, desde la administración local, regional o nacional, pública o privada. Finalmente, un $10 \%$ de la oferta se dirige a un público muy particular, como, por ejemplo, los gerentes o asociados directivos de cooperativas, que desean profundizar su conocimiento y adquirir herramientas para la gestión de sus organizaciones; o bien, a profesionales de ONG, que trabajan en el sector financiero y están interesados en las microfinanzas, en calidad de herramienta para el desarrollo social.

En cuanto a los perfiles de egreso de los programas analizados, se determinó que estos incluyen los ítems relativos a la adquisición de conocimientos y desarrollo de capacidades para la gestión de entidades de la economía social, a través de la dinámica poblacional y sus relaciones con los procesos económicos, sociales, políticos y culturales. Además, tiene que ver con la habilitación para las labores de investigación, reflexión y gestión, en torno a la transformación y el desarrollo económico, social y ambiental local, nacional e internacional.

Finalmente, se observó que el perfil ocupacional de los programas, sometidos al análisis, indica que el campo de acción profesional de los graduados de estas maestrías, en mayor grado, está orientado a las organizaciones sociales, tanto de mercado, como de no mercado, que requieren contar con equipos estratégicos, que poseen la formación específica para tal fin. En menor grado, a roles académicos de docencia e investigación. Por medio de la Figura 6 se resume la información sobre el análisis de los perfiles de ingreso, egreso y de ocupación de los programas de maestría. 


\section{Perfil de ingreso $\rightarrow$ Perfil de salida $\rightarrow$ Perfil ocupacional \\ Profesionales en \\ cualquier área \\ académica. \\ Gestores sociales. \\ Gerentes o asociados \\ en empresas de la \\ economía social.}

\section{Discusión}

La exploración y comparación de los currículos de las maestrías en Economía Social tuvo como propósito caracterizar la oferta de los posgrados ofrecidos en España y Latinoamérica hispanohablante, que forman parte de un proceso de modernización y mejora de la calidad de la educación de alto nivel, respondiendo pertinentemente no solo a las exigencias del mercado laboral, sino también a los retos y necesidades sociales más amplias (Cáceres, 2002; Valle, 2005).

La exploración llevada a cabo permitió determinar que las propuestas curriculares con un enfoque distinto a la enseñanza de la economía convencional, responden a los problemas socioeconómicos en contextos particulares y se comprometen a desarrollar aportes que pueda brindar la economía social a través de modelos alternativos para el desarrollo social y territorial. Para los programas de maestría en Economía Social y afines, ofrecidos en Latinoamérica y España, la comprensión y aplicación de instrumentos propios de política pública y el reforzamiento de capacidades de gestión son fundamentales para el estudio de los fenómenos y procesos sociales que buscan ofrecer aportes sustanciales a las demandas concretas del bienestar social.

Los currículos de estos programas no pierden de vista el hecho de que la economía social no es economía de pobres para pobres, sino que reflejan una visión integrada de un modelo de acumulación basado en la solidaridad y la participación social (Kliksberg, 2002; Sen y Kliksberg, 2009). Sin embargo, no parece existir una definición clara sobre lo que es la economía social y lo que debería enseñarse bajo esta rúbrica: mientras unos programas se enfocan en la comprensión de las entidades económicas (de mercado y de no mercado), otros lo hacen exclusivamente en empresas cooperativas y ONG. Incluso, existen programas que mezclan la conceptualización de la economía social con la de responsabilidad social y la de los territorios socialmente responsables.

Asimismo, mientras algunos programas señalan a las entidades públicas como las responsables del proceso de cambio hacia la nueva economía, otros atribuyen esta responsabilidad a las empresas del sector social, o a las empresas del sector privado, o a una combinación de los tres sectores. De igual forma se presentan discrepancias sobre quiénes son sujetos idóneos para ingresar a estas maestrías $\mathrm{y}$, a pesar de que el objetivo común de los programas es formar agentes de cambio para una sociedad más solidaria, los perfiles ocupacionales se enmarcan en el rol que asumiría el egresado en el mercado laboral exclusivamente. Esas discrepancias y la falta de claridad acerca del enfoque, de los alcances, de los perfiles, entre otros, repercute en la propia oferta académica, la cual en la actualidad se concentra en Sudamérica, aún a pesar de que hay países en los que no se han detectado planes afines, tales como en Chile, Uruguay, Paraguay o Bolivia. 


\section{Conclusiones}

Al efectuar una aproximación exploratoria a los currículos de las maestrías relativas a la Economía Social, ofertadas en el primer semestre de 2016 por las instituciones de educación superior de España y Latinoamérica hispanoparlante, se llega a las siguientes conclusiones, que son de relevancia para los profesionales en economía, entidades de gobierno, académicos, curriculistas y autoridades universitarias.

En primera instancia, se concluye que existe evidencia del interés de la universidad en formar profesionales de alto nivel académico, capaces de brindar opciones alternativas al capitalismo, con una visión real y actualizada sobre aspectos políticos, económicos, sociales y de sostenibilidad financiera y ambiental. Sin embargo, se constata que este interés es limitado, pues la oferta de programas de maestría en Economía Social es relativamente poca y se concentra en los países como España, México, Colombia, Argentina, Ecuador, Costa Rica y Venezuela.

En segundo lugar, se concluye que no existe una convergencia de criterios entre las universidades y programas sobre lo que debería incluirse en el plan de estudios de estas maestrías, dado que los currículos de las maestrías analizadas muestran dos enfoques relacionados, pero distintos: una mayoría se orienta hacia la temática de desarrollo local, aunque también existen otros que se enfocan propiamente en economía social o solidaria.

En tercer lugar, se llega a la conclusión de que, contrario a la mayoría de los posgrados de diversas áreas profesionales, la oferta actual en economía social está dirigida a graduados de cualquier especialidad. A pesar de que la economía social es una rama particular de la economía, no se tiene evidencia sobre programas cuyo sistema de admisión está diseñado para filtrar a los economistas sociales.

Finalmente, se considera que uno de los efectos de la aplicación de los paradigmas económicos convencionales es, cada vez más, el desplazamiento y creciente exclusión de los sectores más vulnerables, por lo cual resulta necesario llevar a cabo una revisión profunda sobre cuál debe ser el rol de la práctica profesional de los gestores de organizaciones sociales y sobre cómo la universidad y el currículo puede contribuir en la generación de competencias orientadas a potenciar y estimular el desarrollo económico inclusivo y solidario. Al respecto, deberían llevarse a cabo esfuerzos regionales que no solo disminuyan las distancias y discrepancias a las que se hizo referencia anteriormente, sino que busquen activamente dar respuesta a necesidades de su realidad socioeconómica desde la formación académica y profesional. $\mathrm{Al}$ respecto, las futuras líneas de investigación en este campo pudieran abordar desde las necesidades de adecuaciones curriculares por tipología de organizaciones, la formulación de contenidos colaborativos, hasta la posibilidad de homologaciones de requisitos entre países para la difusión de planes de estudio e intercambio de docentes y estudiantes que propicien el estudio transdisciplinario en el ámbito de la economía social.

\section{REFERENCIAS}

Aguilar, E. (2016). Economía solidaria y territorio: Complejizando la propuesta de análisis territorial de Coraggio. Polis Revista Latinoamericana, 45, 19-40.

Amin, A.; Cameron, A. y Hudson, R. (2004). Placing the Social Economy. New York: Taylor \& Francis e-Library.

Cáceres, G. (2002). Algunos objetivos estratégicos para el mejoramiento de la educación técnica superior. Boletín $N^{\circ} 3$ MECESUP. Santiago de Chile: Ministerio de Educación.

Chaverri, D. y Sanabria, M. (2010). Estudio comparativo entre los programas que ofrecen tres universidades públicas y siete universidades privadas en la carrera de I y II ciclo de la educación general básica en Costa Rica. Revista Actualidades Investigativas en Educación, 10 (3), 1-33.

Chaves, R. y Monzón, J. (2018): La economía social ante los paradigmas económicos emergentes: innovación social, economía colaborativa, economía circular, responsabilidad social empresarial, economía del bien común, 
empresa social y economía solidaria, CIRIEC-España. Revista de Economía Pública, Social y Cooperativa, 93, 5-50.https://doi.org/10.7203/CIRIEC-E.93.12901.

Chaves, R.; Monzón, J.; Pérez, J. y Radrigán, M. (2013). La economía social en clave internacional: Cuantificación, reconocimiento institucional y visibilidad social en Europa, Iberoamérica y Norte de África. REVESCO, 112, $112-150$.

Coraggio, J. (2011). Economia Social y Solidaria: El trabajo antes que el capital. Quito: FLACSO Ecuador.

Coraggio, J. (2014). La presencia de la economía social y solidaria y su institucionalización en América Latina, UNRISD Occasional Paper: Potential and Limits of Social and Solidarity Economy, No. 7, United Nations Research Institute for Social Development (UNRISD), Geneva.

Fedorov, A. (2016). Closing a Theoretical-Methodological Gap in the Internationalization of Engineering Curricula. International Journal of Engineering Education, 32(5A), 2066-2082. Recuperado de https://bit.ly/2E8hLYL

FLACSO Argentina. (2019). Desarrollo local, territorial y economía social: Presentación. Recuperado de https://bit.l $\mathrm{y} / 2 \mathrm{PeC} 4 \mathrm{Ig}$

FLACSO México. (s.f.). Maestría en población y desarrollo: Presentación. Recuperado de https://bit.ly/1NfFnqU

García, A. y Ballabrera, J. (2015). Energy and mineral peaks, and a future steady state economy. Technological Forecasting \& Social Change, 90 (B), 587-598

Gimeno, J. (1991). El currículum: una reflexión sobre la práctica. Madrid: Ediciones Morata, S. L

Gómez, V. (2013). Acercamiento a las prácticas de la economía social, la economía solidaria y la Economía del Bien Común, ¿qué nos ofrecen? BARATARIA Revista Castellano-Manchega de Ciencias Sociales, 15, 112-124.

Gómez, V.y Gómez, R. (2014). Herramientas de la economía del bien común para la transformación económica, social y política. Deusto Estudios Cooperativos, 5, 13-41.

Guin, A. y Vidal, M. (2015). Enseñanza de la economía en la Pontificia Universidad Católica de Chile: un análisis comparativo. Estudios Nueva Economia, 5, 47-55.

Kemmis, S. (1998). El currículum: más allá de la teoria de la reproducción. Madrid: Ediciones Morata, S. L.

Kliksberg, B. (2002). El capital social: Dimensión olvidada del desarrollo. Caracas: Universidad Metropolitana.

Lora, E. y Ñopo, H. (2009). La formación de los economistas en América Latina. Revista de Análisis Económico, 24(2), 65-93.

Molloy, A., McFeeley, C. y Connolly, E. (1999). Building a Social Economy for the New Millennium. Derry: Guildhall Press.

Pedreño, J. (2017). Reflexiones, sinergias y clarificación sobre nuevos conceptos y modelos: economía social, empresa social, emprendimiento social, economía del bien común, economía solidaria y economía colaborativa. Revista Española del Tercer Sector, 35, 45-72.

Pérez de Mendiguren, J., Etxezarreta, E. y Guridi, L. (2008, marzo). ¿De qué hablamos cuando hablamos de economía social y solidaria? Concepto y nociones afines. Ponencia presentada en la XI Jornada de Economía Crítica, Bilbao, España. Recuperado de https://bit.ly/2scVy3u

Piketty, T. (2014). El capital en el siglo XXI. Madrid: Fondo de Cultura Económica de España.

Pontificia Universidad Católica del Ecuador. (2014). Maestría en gestión del desarrollo local comunitario. Recuperado de https://bit.ly/2VjZPjD

Samuelson, P. y Nordhaus, W. (2001). Macroeconomía. México: Mc-Graw Hill.

Sen, A y Kliksberg, B. (2009). Primero la gente. Barcelona: Deusto.

Stenhouse, L. (2003). Investigación y desarrollo del curriculum. Madrid: Ediciones Morata, S. L.

Tomás, J. (1997). La economía social en un mundo de transformación. Revista de Economía Pública, Social y Cooperativa, 25, 83-115.

Universidad Central de Venezuela. (2016). Maestría en planificación del desarrollo. Recuperado de https://bit.ly/2Q $0 \mathrm{FaQI}$ 
Dyalá de la O, et al. Exploración sobre la oferta de los posgrados en Economía Social en los país...

Universidad Nacional de General Sarmiento (2019). Maestría en economía social: Descripción. Recuperado de https: //bit.ly/2OwnNtE

Valle, M. (2005). Base de comparación de mallas curriculares de carreras de ingeniería civil. Revista Iberoamericana de Educación, 36 (5), 1-13.

\section{Notas}

[1] Los nombres, prácticas, criterios y sentidos a los que se refiere Coraggio (2011) son los siguientes: economía social, economía solidaria, economía de la solidaridad, economía social y solidaria, economía comunitaria, economía popular, otra economía; emprendimientos asociativos, empresas recuperadas, empresas sociales, cooperativas, cadenas de valor, asociaciones de productores, asociaciones de consumidores, redes de ayuda mutua, fondos de crédito rotatorios, microcrédito, redes de trueque, cambalaches, ferias populares, producción para el autoconsumo, huertos familiares y/o comunitarios, migraciones y remesas; eficiencia, sostenibilidad, subsidio, trabajo genuino, trabajo autogestionado, territorio, desarrollo endógeno; desarrollo y equidad, democratización de la economía, consumo responsable, comercio justo, justicia social, solidaridad, reciprocidad, reproducción ampliada de la vida, Buen Vivir, Vivir Bien.

\section{BY-NC-ND}

